\title{
Kinetics of a Solid-Solid Charge Transfer Reaction
}

\author{
Ying Pan ${ }^{1}$, Dimos Poulikakos ${ }^{2}$, Nate Cira $^{1}$, and Ye Tao ${ }^{1 *}$ \\ 1) Rowland Institute at Harvard, Cambridge MA, USA \\ 2) ETH Zurich, Zurich, Switzerland \\ ${ }^{*}$ Corresponding and presenting author: E-mail tao@,rowland.harvard.edu
}

We present a detailed analysis of the kinetics of electron transfer at a solid-solid interface. The study was enabled by measuring the conductance of ultra-thin $(<50 \mathrm{~nm})$, fully suspended single-crystalline silicon nanowires with several well-defined surface chemistries [1]. The measurement had a dynamic range across 7 orders of magnitude with a detection sensitivity of order single event over a $10^{3} \mu \mathrm{m}^{2}$ reaction area. We find that classic, first-order process $[2,3]$ is unable to capture the reaction progress and propose a new kinetic model based on a continuum of reactant geometries inherently present at typical solid-solid interfaces. The new model captures the kinetic manifestation of heterogeneity in a single, global rate constant. Quantitative agreement with data and an analysis of the parameters suggest that this model may be generally applicable to charge transfer at solid-solid interfaces.

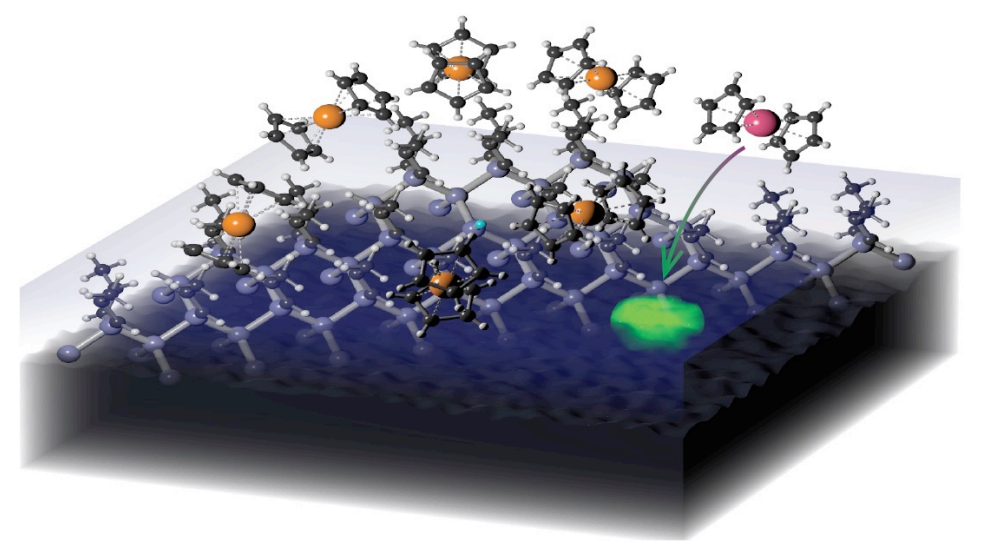

Figure 1. Illustration of a nanoscale charge transfer reaction interface. Cobaltocene molecules (orange) on top of a 3-carbon monolayer covalently attached to atomically-rough $\mathrm{Si}(100)$ (purple). One positively-charged cobaltocenium ions (crimson sphere) transferred an electron (green) to the lattice. A Si(111) microfacet is depicted with a silicon-fluorine (cyan) defect. Such surface defects result from the fabrication of nanoscale materials and devices.

\section{References.}

[1] Y. Tao, R. Hauert, and C. L. Degen, ACS Appl. Mater. Interfaces 8, 13157-13165 (2016).

[2] C. E. D. Chidsey, Science 251, 919-922 (1991).

[3] D. M. Adams, L. Brus, C. E. D. Chidsey, S. Creager, C. Creutz, C. R. Kagan, P. V. Kamat, M. Lieberman, S. Lindsay, R. A. Marcus, R. M. Metzger, M. E. Michel-Beyerle, J. R. Miller, M. D. Newton, D. R. Rolison, O. Sankey, K. S. Schanze, J. Yardley, and X. Zhu, Phys. Chem. B 107, 6668-6697 (2003). 